\title{
Neutrophil-lymphocyte ratio as a prognostic factor for stereotactic body radiotherapy treatment of metastatic liver tumors
}

\author{
Xuyun Xie ${ }^{1}$, Jingjing Shan ${ }^{1}$, Xuan Zhou ${ }^{2}$, Hai Liu ${ }^{1}$, Xiaonan Sun ${ }^{1}$ \\ ${ }^{1}$ Department of Radiation Oncology, ${ }^{2}$ Department of Nursing, Sir Run Run Shaw Hospital, School of Medicine, Zhejiang University, Hangzhou, \\ China \\ Contributions: (I) Conception and design: X Sun; (II) Administrative support: X Sun; (III) Provision of study materials or patients: H Liu; (IV) \\ Collection and assembly of data: X Xie, J Shan, X Zhou; (V) Data analysis and interpretation: X Xie; (VI) Manuscript writing: All authors; (VII) Final \\ approval of manuscript: All authors. \\ Correspondence to: Prof. Xiaonan Sun. Department of Radiation Oncology, Sir Run Run Shaw Hospital, School of Medicine, Zhejiang University, 3 \\ Qingchun East Road, Hangzhou, China. Email: sunxiaonan@zju.edu.cn.
}

Background: The neutrophil-lymphocyte ratio (NLR) has been proposed as an indicator of systemic inflammatory response and may be able to predict clinical outcomes in patients with malignant solid tumors. However, the relationship between NLR and the prognosis of liver metastasis after stereotactic body radiotherapy (SBRT) is not well established.

Methods: Patients with liver metastatic tumors who underwent SBRT in our center between 2015 and 2018 were enrolled, and their data were retrospectively analyzed. The patient' baseline total white blood cell (WBC), neutrophil, lymphocyte, and platelet counts were recorded, along with their mean platelet volume (MPV) and red blood cell distribution width (RDW). The cut-off values for NLR and platelet-lymphocyte ratio (PLR) were determined by receiver operating characteristic (ROC) curves. Local control (LC) and overall survival (OS) were calculated using the Kaplan-Meier method. The Cox proportional hazards model was used to study significant variable line and factors associated with OS.

Results: A total of 65 patients with 82 lesions were enrolled. The median duration follow-up was 21 months. The local control (LC) rate was $76.8 \%$, and the 1 - and 2 -year OS rates were $70.7 \%$ and $46.1 \%$, respectively. The cut-off values of NLR and PLR were 3.16 and 201.33, respectively. Multivariate analysis identified the NLR cut-off value as a potential independent indicator of inferior OS $[\mathrm{P}=0.005, \mathrm{HR}=3.317$ (95\% CI, 1.433-7.678)]. Biological effective dose (BED) was also associated with $\mathrm{OS}(\mathrm{P}=0.035, \mathrm{HR}=0.984$ (95\% CI, 0.969-0.999)].

Conclusions: NLR showed a strong association with LC and OS, which could serve as a predictive guide in the treatment of liver metastasis with SRBT. BED is also an independent factor affecting OS.

Keywords: Liver metastatic tumor; neutrophil-lymphocyte ratio (NLR); biological effective dose; stereotactic body radiotherapy (SBRT)

Submitted Mar 04, 2020. Accepted for publication Aug 07, 2020.

doi: $10.21037 /$ tcr-20-1335a

View this article at: http://dx.doi.org/10.21037/tcr-20-1335a

\section{Introduction}

Due to its involvement in hematogenous metastasis, it is the primary cause of death in patients with malignant tumors (1). Surgical resection is the standard recommended local therapy for patients, but its therapeutic efficacy is limited (2). Aggressive treatments including stereotactic body radiotherapy (SBRT), thermal ablation, and transcatheter arterial chemoembolization have improved the prognosis in suitable patients with hepatic metastases. An increasing number of studies have reported that patients with liver metastases who cannot tolerate or 
refuse surgery may benefit from SBRT (3). To minimize patient discomfort and administer effective and targeted treatments, prognostic factors are urgently required as a guide to personalized therapy.

Inflammatory responses play specific roles at various stages of tumor development, including initiation, promotion, malignant conversion, invasion, and metastasis. Inflammation also affects immune surveillance and responses to therapy (4). Hematological parameters, including neutrophil-lymphocyte ratio (NLR), plateletlymphocyte ratio (PLR), mean platelet volume (MPV) and red blood cell distribution width (RDW), are widely used in the diagnosis of systemic chronic inflammation due to the ease and low costs associated with routine blood tests. These prognostic markers have been considered as important prognostic indicators in patients with malignant solid tumors (4-9). Dermot demonstrated that by changing the tumor microenvironment, chronic inflammation plays an important role in non-small cell lung cancer (5). Dr. Luo determined that the change of NLR may serve as a potential biomarker for OS in patients with advanced pancreatic cancer undergoing chemotherapy (8).

However, these studies primarily focused on surgery, chemotherapy, and conventional radiotherapy. The benefits of SBRT, however, have not been investigated. In this study, we examined the relationship between systemic inflammatory biomarkers and the prognosis of patients with liver metastasis who underwent SBRT. We present the following article in accordance with the STROBE reporting checklist (available at http://dx.doi.org/10.21037/tcr-201335a).

\section{Methods}

\section{Patient selection}

The data of 136 patients with liver metastasis, who received SBRT at Sir Run Run Shaw Hospital between July, 2015 and January, 2018, retrospectively analyzed. The inclusion criteria were as follows: (I) two kinds of imaging examination indicating liver metastasis, usually enhanced computed tomography (CT) of the upper abdomen and liver magnetic resonance examination (MR); (II) an Eastern Cooperative Oncology Group (ECOG) Performance Status score of $0-2$, and Child-Pugh A; (III) complete blood count (CBC) obtained a week before radiotherapy; (IV) complete follow-up. Patients meeting any of the following criteria were excluded: (I) evidence of infection before SBRT, including, but not limited to, as high fever, respiratory infection, or urinary infection; (II) active hemorrhage, blood transfusion within 3 months, chronic infection, autoimmune disease, or steroid use affecting blood routine counts; (III) loss to follow-up, non-neoplastic death, or under-regulated treatment. The study was conducted in accordance with the Declaration of Helsinki (as was revised in 2013) . The study was approved by ethics committee of Sir Run Run Shaw hospital, School of Medicine, Zhejiang University (NO.: 20190927-1). The requirement for individual consent was waived due to the retrospective nature of this study. No patient-identifiable information was utilized for this study.

\section{Treatments}

SBRT was performed according to our institutional standard. Each patient was immobilized in the supine position, supported with a customized, vacuum cushion (R7619NLB, Klarity). Then, four-dimensional computed tomography (4D-CT) localization (Real-Time Position Management, Siemens CT, SAMATOM Definition AS Open) was performed. The patients' respiratory motion was managed with abdominal compression. The 4D-CT images were reconstructed using a phase-based binning method with 10 respiratory phases. The gross tumor volume (GTV) was delineated at ten phases, assisted by contrast-enhanced magnetic resonance imaging (MRI), and the internal target volume (ITV) was acquired by merging the GTVs of 10 respiratory phases. The planning target volume (PTV) was generated from the ITV by adding an overall isotropic margin of $5 \mathrm{~mm}$. Contouring and treatment planning were developed using a three-dimensional radiation therapy planning system (Eclipse V11.0; Varian Medical Systems). The prescribed dose was 24-60 Gy and delivered in 3-5 fractions. SBRT was routinely administered every other day for the duration of 2 weeks. Follow-up examinations consisted of CT and MRI examinations every 3 months for 2 years, then at half-yearly for the 3 years after that.

\section{Biomarkers of systemic inflammation}

This study investigated markers of systemic inflammation by recording the patients' WBC, neutrophil, lymphocyte, and platelet counts, as well as their RDW and MPV,1 week before the start of SBRT. Blood samples were collected, processed, and analyzed at our institution.

$$
\left(\mathrm{NLR}=\frac{\text { neutrophil count }}{\text { lymphocyte count }} ; \mathrm{PLR}=\frac{\text { platelet count }}{\text { lymphocyte count }}\right)
$$




\section{Therapeutic efficacy}

(I) Short term treatment outcomes: local control (LC) was evaluated 1 month after SBRT by liver magnetic resonance (MR) examination, according to the modified Response Evaluation and Criteria in Solid Tumors (RECIST) criteria. LC was defined as complete response (CR), partial response (PR), stable disease (SD) of the treated lesions.

(II) Long term treatment outcomes: telephone contact and visits were undertaken to record the patients' information. The follow-up period was defined as the time from the first date of SBRT to January 13, 2020. OS was defined as the time from the start of SBRT to the date of death or the last follow-up.

\section{Statistical analysis}

LC and OS were calculated using the Kaplan-Meier method, with the log-rank test used to detect potential differences. OS was analyzed for each patient, while LC was calculated for each lesion. Receiver operating characteristic (ROC) curve analysis was used to identify the valuable index of systemic inflammation for LC, determining the cut-off value at the same time. Univariate and multivariate Cox proportional hazards regression analysis was performed to identify factors that were significantly associated with OS. To determine the correlation between systemic inflammation biomarkers and clinical features, the MannWhitney $U$ test was used to compare continuous variables, while chi-square analysis was performed to compare categorical variables. A $\mathrm{P}$ value of $<0.05$ was considered to be statistically significant. All analyses were conducted using SPSS version 20.0 (IBM Corporation, Armonk, NY, USA).

The study was conducted in accordance with the Declaration of Helsinki (as was revised in 2013). The study was approved by ethics committee of Sir Run Run Shaw hospital, School of Medicine, Zhejiang University (NO.: 20190927-1).

\section{Results}

\section{Patient characteristics}

This study included 65 patients (42 males and 23 females) with a combined total of 82 lesions (53 single lesions and 29 multi-lesions). The patients' clinical characteristics are shown in Table 1. The selection process is shown in Figure 1.The primary tumor types identified included colorectal adenocarcinoma (CRC), which was the most common type $(46.15 \%)$, followed by pancreatic $(21.54 \%)$, lung $(10.77 \%)$, gastroesophageal $(10.77 \%)$, gynecologic $(3.08 \%)$ and other (1 ureteral, 1 malignant melanoma, 1 leiomyosarcoma, 1 nasopharyngeal, and 1 breast) tumors. All primary cancers were subjected to radical treatments, such as resection and radio-chemotherapy and were stable during liver metastasis. The majority of patients (70.77\%) prior treatment naive, while the remaining patients (43.08\%) had undergone sequential therapy. Twenty-two patients did not receive subsequent systematic therapy due to chemotherapy intolerance, financial reasons, or a shortage of effective treatment at that time. These patients underwent close monitoring during the follow-up period. The median SBRT dose was 45 Gy (24-60 Gy) delivered in a median of 5 fractions (range, $3-5$ fractions).

\section{Predictive value of inflammation markers and correlation analysis}

Table 2 lists the areas under ROC curves (AUC) of inflammatory markers. NLR displayed the highest AUC $(0.821, \mathrm{P}=0.010<0.05)$, followed by PLR $(0.808$, $\mathrm{P}=0.014<0.05)$, RDW $(0.669, \mathrm{P}=0.174>0.05)$, and MPV (0.596, $\mathrm{P}=0.441>0.05)$. NLR and PLR displayed higher diagnostic values. The optimal cutoff points of NLR and PLR were determined to be 3.16 and 201.33, respectively, based on sensitivity and specificity shown in the ROC curve (Figure 2). As illustrated in Table 3, no clinical features achieved statistical significance $(\mathrm{P}<0.05)$, in chi-square and Mann-Whitney $\mathrm{U}$ analyses, indicating that NLR and PLR were independent of these clinical features.

\section{Survival analyses}

Of the 82 lesions treated, 63 achieved PR, CR, or SD after SBRT, with an LC rate of $76.8 \%$. The 1- and 2-year OS rates were $70.7 \%$ and $46.1 \%$, respectively, with a mean follow-up duration of 21 months (range, 1.7-34 months). We analyzed the four groups (LC based on NLR; LC based on PLR; OS based on NLR; OS based on PLR) by KaplanMeier survival analysis and noted a correlation between high NLR and PLR values and significantly decreased LC and OS (Figure 3).

Based on univariate analysis for OS (Table 3), NLR $[\mathrm{P}=0.000$, hazard ratio $(\mathrm{HR})=7.907,95 \%$ confidence interval 
Table 1 Characteristics of the subjects

\begin{tabular}{|c|c|}
\hline Variable & Frequency \\
\hline Patients & 65 \\
\hline Lesions & 82 \\
\hline \multicolumn{2}{|l|}{ Gender, n (\%) } \\
\hline Male & $42(64.62)$ \\
\hline Female & $23(35.38)$ \\
\hline Median age (range) in years & $62.0(32-90)$ \\
\hline \multicolumn{2}{|l|}{ Primary tumor, $\mathrm{n}(\%)$} \\
\hline Colorectal & $30(46.15)$ \\
\hline Lung & $7(10.77)$ \\
\hline Gastroesophageal & $7(10.77)$ \\
\hline Pancreas & $14(21.54)$ \\
\hline Gynecological & $2(3.08)$ \\
\hline Other & 5 (7.69) \\
\hline \multicolumn{2}{|l|}{ Prior treatment, n (\%) } \\
\hline Chemotherapy & $14(21.54)$ \\
\hline Radio frequency ablation (RFA) & $1(1.54)$ \\
\hline TACE & $4(6.15)$ \\
\hline target-treatment & $6(9.23)$ \\
\hline No prior treatment & $46(70.77)$ \\
\hline \multicolumn{2}{|l|}{ Sequential therapy, n (\%) } \\
\hline Chemotherapy & $19(29.23)$ \\
\hline Surgery & $4(6.15)$ \\
\hline TACE & $3(4.62)$ \\
\hline target-treatment & $7(10.77)$ \\
\hline Radio frequency ablation (RFA) & $2(3.08)$ \\
\hline Immunotherapy & $1(1.54)$ \\
\hline No treatment & 37 (56.92) \\
\hline Median dose (range) in Gy & $45.0(24-60)$ \\
\hline Median BED (range) in Gy & $100.0(43.2-129.375)$ \\
\hline Median number of fractions (range) & $5.0(3.0-5.0)$ \\
\hline Median dose per fraction (range) in Gy & $10.0(6-12.5)$ \\
\hline
\end{tabular}

TACE, transcatheter arterial chemoembolization; BED, biological effective dose.

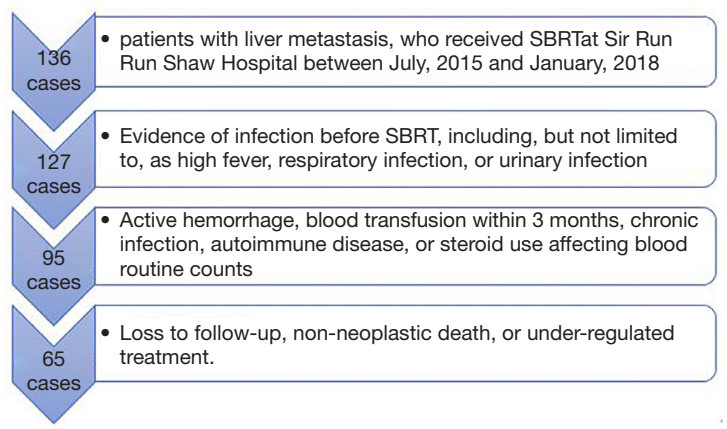

Figure 1 A flow diagram for cases selection.

Table 2 AUC of laboratory hematological parameter for predicting LC

\begin{tabular}{lccc}
\hline Index tests & AUC & $95 \% \mathrm{Cl}$ & $\mathrm{P}$ \\
\hline NLR & 0.821 & $0.723-0.918$ & 0.010 \\
PLR & 0.808 & $0.663-0.952$ & 0.014 \\
RDW & 0.669 & $0.446-0.893$ & 0.174 \\
MPV & 0.596 & $0.345-0.847$ & 0.441 \\
\hline
\end{tabular}

TACE, transcatheter arterial chemoembolization; NLR, neutrophillymphocyte ratio; PLR, platelet-lymphocyte ratio; RDW, red blood cell distribution width; MPV, mean platelet volume.

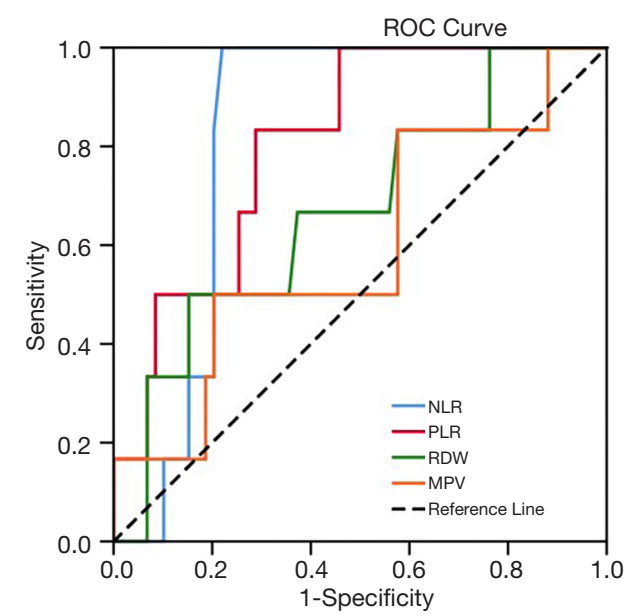

Figure 2 The ROC curve of NLR (blue); the ROC curve of PLR (red); the ROC curve of RDW (green); the ROC curve of MPV (orange). ROC, receiver operating characteristic; NLR, neutrophillymphocyte ratio; PLR, platelet-lymphocyte ratio; RDW, red blood cell distribution width; MPV, mean platelet volume. 
Table 3 Correlations between hematological parameters and clinic features

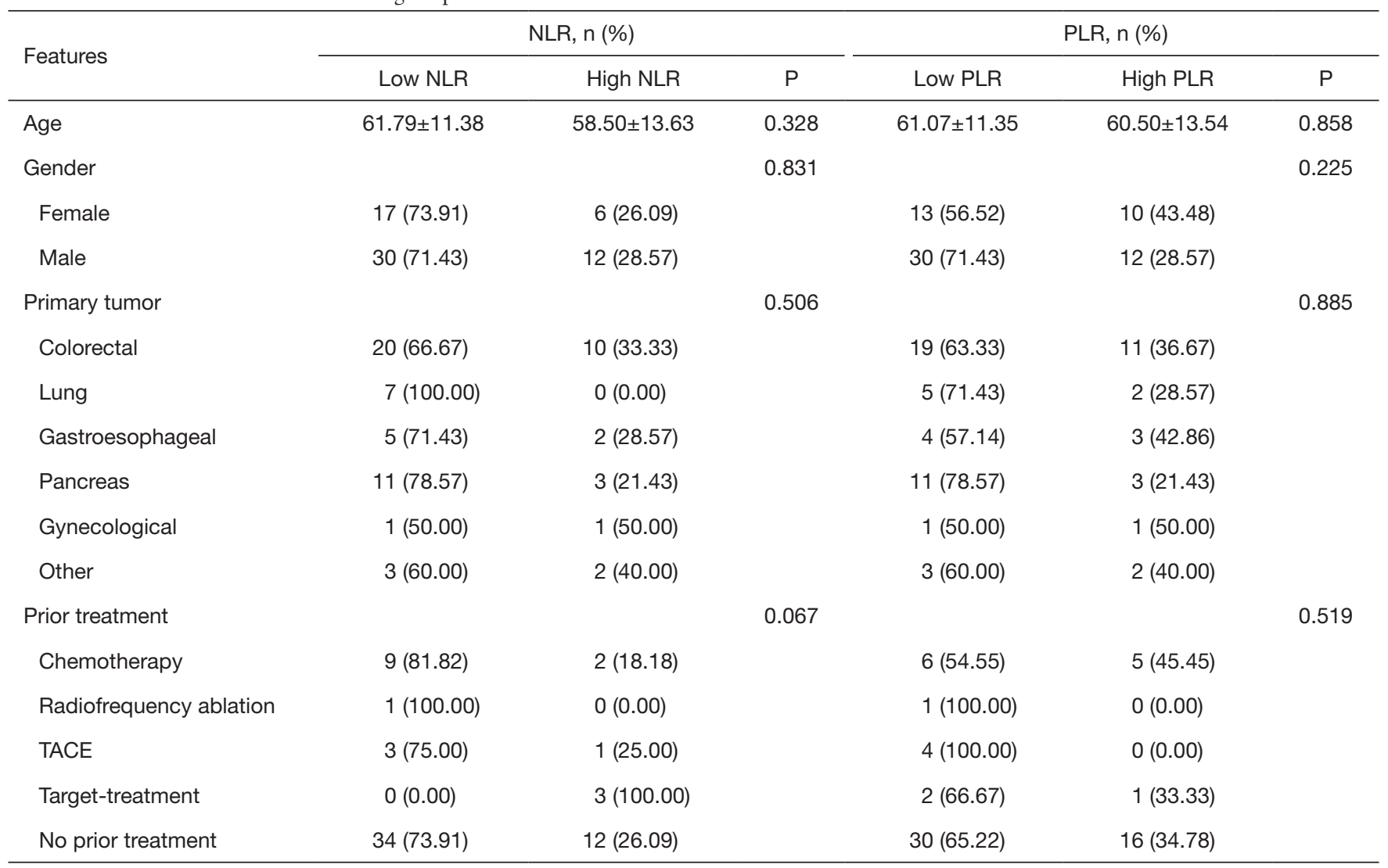

TACE, transcatheter arterial chemoembolization; NLR, neutrophil-lymphocyte ratio; PLR, platelet-lymphocyte ratio; RDW, red blood cell distribution width; MPV, mean platelet volume.

(CI): 3.708-16.862], PLR $(\mathrm{P}=0.035, \mathrm{HR}=2.175,95 \%$ CI: $1.057-4.476), \mathrm{RDW}(\mathrm{P}=0.002, \mathrm{HR}=1.174,95 \% \mathrm{CI}$ : 1.062-1.298), BED ( $\mathrm{P}=0.001, \mathrm{HR}=0.976$, 95\% CI: $0.962-$ $0.990)$, and other primary tumor types were relevant to OS. Multivariate analysis identified NLR $(\mathrm{P}=0.005, \mathrm{HR}=3.317$, 95\% CI: $1.433-7.678)$ and BED $(\mathrm{P}=0.035, \mathrm{HR}=0.984,95 \%$ CI: 0.969-0.999) as independent factors for OS.

\section{Discussion}

This retrospective study demonstrates favorable median OS (21 months) and durable LC (median, 21 months) in a cohort of patients with liver metastasis who received SBRT. The overall LC rate was $76.8 \%$, and the 1 - and 2 -year OS rates were $70.7 \%$ and $46.1 \%$, respectively. These results are with other SBRT reports on liver metastases, in which the 1-year LC rates ranged from $71-100 \%$ and the 2 -year rates from $30-66 \%(10-13)$. Our study suggested that a higher BED delivered enhanced OS. A multi-institutional study investigating the efficacy of SBRT for treating liver metastasis revealed a correlation between elevated BED with increased LC and OS (3). This suggests a need for further investigations into the importance of BED and its association with LC and OS Moreover, our study did not identify any correlation between total dose, dose per fraction, and outcomes, which is consistent with several clinical trials recently reported (12).

Our findings illustrate that NLR, as a biomarker of systemic inflammation, was linked to the outcomes of patients with hepatic metastatic tumors who underwent SBRT. NLR values of $\leq 3.16$ considered to be an independent prognostic factor for SBRT suitability. Meanwhile, this study found no association between NLR values and other clinical features of hepatic metastatic tumors treated with SBRT.

Inflammation is reported in every step of tumorigenesis, from initiation to tumor promotion to eventual metastatic progression (4). An elevated NLR was an independent 

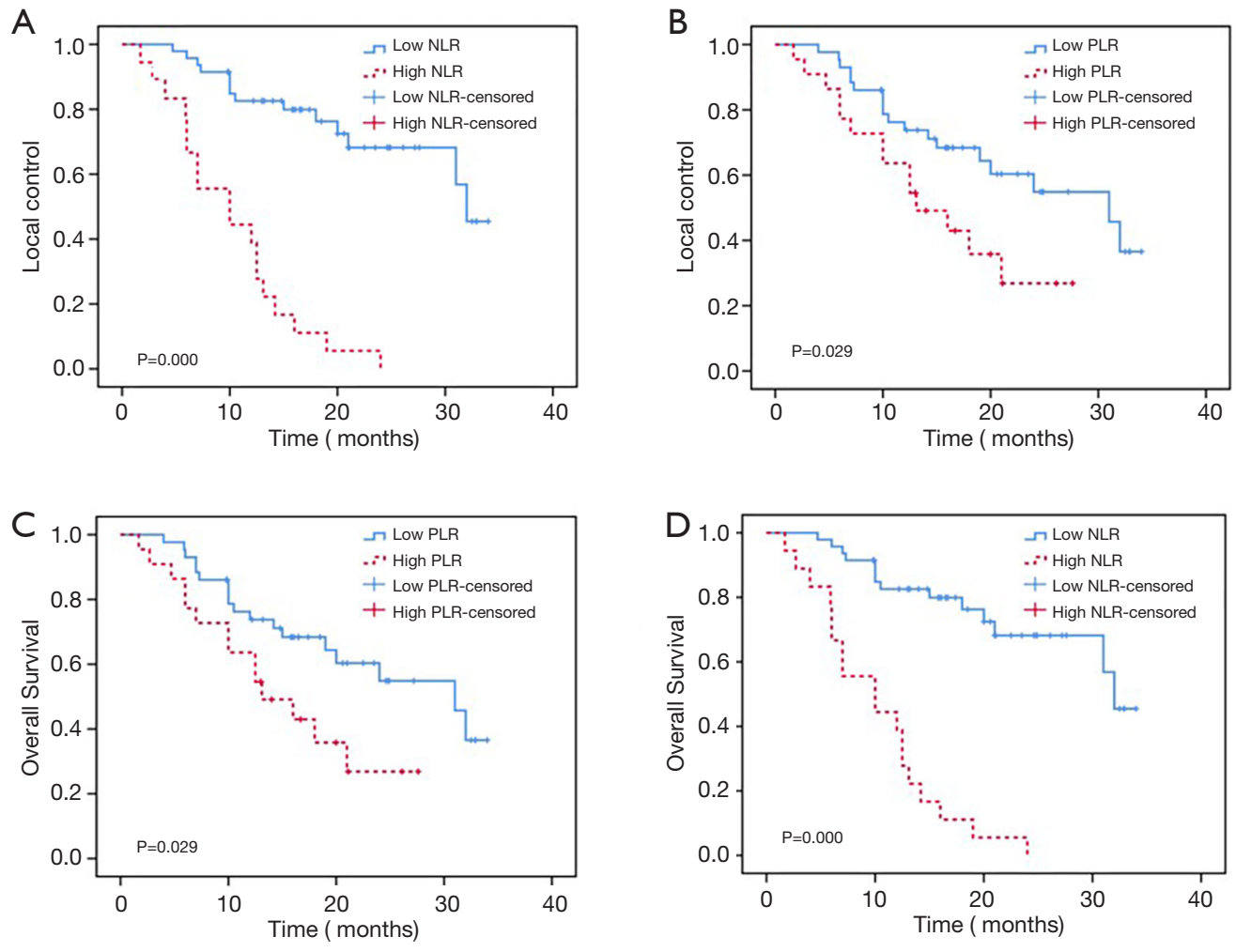

Figure 3 Outcome in patients with liver metastatic tumors. (A) Local control based on neutrophil-to-lymphocyte ratio. Solid blue line: NLR $\leq 3.16$, dashed red line: NLR $>3.16$. (B) Local control based on platelet-to-lymphocyte ratio. Solid blue line: PLR $\leq 201.33$, dashed red line: PLR >201.33. (C) Overall survival based on neutrophil-to-lymphocyte ratio. Solid blue line: NLR $\leq 3.16$, dashed red line: NLR $>3.16$. (D) Overall survival based on platelet-to lymphocyte-ratio. Solid blue line: PLR $\leq 201.33$, dashed red line: PLR $>201.33$. NLR, neutrophillymphocyte ratio; PLR, platelet-lymphocyte ratio.

marker for poor prognosis and decreased OS in most patients with solid tumors. Kabarriti et al. found that high NLR was also associated with poor OS in a mouse model (14). Numerous studies have shown that NLR may be a predictor of mortality in gastric cancer (15), liver (16), and lung cancer (17) patients who undergo conventional radiotherapy. However, SBRT delivers a large dose of radiation to the tumor and is potentially more effective than conventional radiotherapy.

The mechanism of NLR in tumor progression is still unclear. Circulating neutrophils, infiltrate tumors produce factors (e.g., ELA2, OSM, and S100A8/9) that promote tumor cell proliferation, survival, and resistance to antitumor therapy. They are also known to induce epithelialmesenchymal transition (EMT) of tumor cells and support their migration machinery, such as MMP9, S100A8/9, Bv8, and leukotrienes. Moreover, neutrophils have been shown to be key effector cells in innate immunity by impairing T-cell response, inducing T-cell death, and recruiting regulatory $\mathrm{T}$ cells, which play an important role in cancer development and progression (18). Furthermore, a low lymphocyte microenvironment weakens the immune surveillance of tumors, and promotes tumor proliferation and migration (19). Therefore, increased of NLR values suggested enhanced inflammation and weakening of anti-tumor immunity, meaning NLR could be used as a pre-treatment measure for selecting patients with liver metastasis who may benefit from SBRT, combined with immunotherapy.

Radiosensitivity index (RSI) is believed to be closely associated with the nature and biological behavior of the primary tumor. In this study, patients with liver metastases from different primary cancers were enrolled. CRC was the most common primary tumor type, followed by pancreatic, lung, gastroesophageal, gynecologic, and other tumors. Such composition corresponded to the 
proportion of primary tumors in most existing studies on RSI (20). A high dose per fraction and a different delivery pattern lowered the duration of SBRT, thereby limiting the potential for sublethal damage repair compared to conventional radiotherapy (CRT). Thus, the RSI of different primary tumors that receive SBRT is different to that of primary tumors that receive CRT. Compared to SBRT, the influence is always smaller than CRT. K.A. Ahmed et al. identified a multigene expression index for tumor RSI with validation in multiple cohorts; the median RSI for skin colorectal, stomach, pancreatic, lung, and breast cancer with liver metastases was $0.43,0.43,0.42,0.35$, and 0.34 , respectively $\mathrm{P}=0.0002$ (21). Most primary tumors included in this study displayed no significant difference in RSI. This may due to the distinctive biology of the liver, which is focused on interactions between disseminated tumor cells and the unique resident cell populations of the liver (22). The resident cell populations of the liver (hepatocytes, liver sinusoidal endothelial cells, Kupffer cells, and hepatic stellate cells) exhibit tumoricidal (apoptosis, phagocytosis, and endocytosis) and/or tumor-promoting activities (extravasation, arrest, colonization, proliferation, angiogenesis, and immunosuppression) in the hepatic metastatic microenvironment. Activities are mediated via soluble signaling factors, direct receptor-mediated cellcell or cell-ECM contacts, and proteolytic enzymes. This mechanism may cause the hemodynamic features and a unique microenvironment of the liver more important than the location of the primary tumor. Moreover, all primary cancers in this study had received radical treatments, such as resection and radio-chemotherapy, further justifying our focus on liver metastasis.

However, the selection of critical NLR values is influenced by the number of samples, research methods, biological characteristics and the location of tumors (23). There are still some shortcomings in this study, such as the small sample size and the bias associated with retrospective studies. To further test and verify the viability of our findings, further prospective randomized clinical trials with large samples and suitable patients are necessary. Additionally, the mechanism of NLR in tumor underwent SBRT progression is still unclear, with further clarifications to be obtained through animal model investigations.

\section{Conclusions}

In conclusion, our data suggest that NLR and BED were associated with OS in metastatic liver patients who received
SBRT. The NLR could be considered as an independent prognostic indicator for clinical cancer treatment tailored for individual patients. Within the tolerable range of patients, an increased BED may achieve an improved therapeutic effect.

\section{Acknowledgments}

Funding: This work was supported in part by grants from the Medical and Health Research Program of Zhejiang Province (No. 2018RC049).

\section{Footnote}

Reporting Checklist: The authors have completed the STROBE reporting checklist. Available at: http://dx.doi. org/10.21037/tcr-20-1335a

Data Sharing Statement: Available at http://dx.doi. org/10.21037/tcr-20-1335a

Peer Review File: Available at http://dx.doi.org/10.21037/tcr20-1335a

Conflicts of Interest: All authors have completed the ICMJE uniform disclosure form (available at: http://dx.doi. org/10.21037/tcr-20-1335a). The authors have no conflicts of interest to declare.

Ethical Statement: The authors are accountable for all aspects of the work in ensuring that questions related to the accuracy or integrity of any part of the work are appropriately investigated and resolved. The study was conducted in accordance with the Declaration of Helsinki (as revised in 2013). The study was approved by ethics committee of Sir Run Run Shaw hospital, School of Medicine, Zhejiang University (NO.: 20190927-1). The need requirement for individual consent was waived due to the retrospective nature of this study. No patientidentifiable information was utilized for this study.

Open Access Statement: This is an Open Access article distributed in accordance with the Creative Commons Attribution-NonCommercial-NoDerivs 4.0 International License (CC BY-NC-ND 4.0), which permits the noncommercial replication and distribution of the article with the strict proviso that no changes or edits are made and the original work is properly cited (including links to both the 
formal publication through the relevant DOI and the license). See: https://creativecommons.org/licenses/by-nc-nd/4.0/.

\section{References}

1. Chen W, Sun K, Zheng R, et al. Cancer incidence and mortality in China, 2014. Chin J Cancer Res 2018;30:1-12.

2. Nordlinger B, Vauthey JN, Poston G, et al. The timing of chemotherapy and surgery for the treatment of colorectal liver metastases. Clin Colorectal Cancer 2010;9:212-8.

3. Mahadevan A, Blanck O, Lanciano R, et al. Stereotactic Body Radiotherapy (SBRT) for liver metastasis - clinical outcomes from the international multi-institutional RSSearch ${ }^{\circledR}$ Patient Registry. Radiat Oncol 2018;13:26.

4. Grivennikov SI, Greten FR, Karin M. Immunity, inflammation, and cancer. Cell 2010;140:883-99.

5. O'Callaghan DS, O'Donnell D, O'Connell F, et al. The role of inflammation in the pathogenesis of non-small cell lung cancer. J Thorac Oncol 2010;5:2024-36.

6. Aggarwal BB, Vijayalekshmi RV, Sung B. Targeting inflammatory pathways for prevention and therapy of cancer: short-term friend, long-term foe. Clin Cancer Res 2009;15:425-30.

7. van Verschuer VM, Hooning MJ, van Baare-Georgieva $\mathrm{RD}$, et al. Tumor-associated inflammation as a potential prognostic tool in BRCA1/2-associated breast cancer. Hum Pathol 2015;46:182-90.

8. Luo G, Guo M, Liu Z, et al. Blood neutrophil-lymphocyte ratio predicts survival in patients with advanced pancreatic cancer treated with chemotherapy. Ann Surg Oncol 2015;22:670-6.

9. Shalapour S, Karin M. Immunity, inflammation, and cancer: an eternal fight between good and evil. J Clin Invest 2015;125:3347-55.

10. Blomgren H, Lax I, Näslund I, et al. Stereotactic high dose fraction radiation therapy of extracranial tumors using an accelerator. Clinical experience of the first thirty-one patients. Acta Oncol 1995;34:861-70.

11. Herfarth KK, Debus J, Lohr F, et al. Stereotactic singledose radiation therapy of liver tumors: results of a phase $\mathrm{I} /$ II trial. J Clin Oncol 2001;19:164-70.

12. Rusthoven KE, Kavanagh BD, Cardenes H, et al. Multiinstitutional phase I/II trial of stereotactic body radiation therapy for liver metastases. J Clin Oncol 2009;27:1572-8.

13. Lee MT, Kim JJ, Dinniwell R, et al. Phase I study of individualized stereotactic body radiotherapy of liver metastases. J Clin Oncol 2009;27:1585-91.

14. Kabarriti R, Zhang Y, Savage T, et al. NLR as a Prognostic Factor in Solid Tumors and in a Transplantable Solid Tumor Mouse Model. International Journal of Radiation Oncology Biology Physics 2018;102:e172.

15. Mohri Y, Tanaka K, Ohi M, et al. Prognostic significance of host- and tumor-related factors in patients with gastric cancer. World J Surg 2010;34:285-90.

16. Lai Q, Castro Santa E, Rico Juri JM, et al. Neutrophil and platelet-to-lymphocyte ratio as new predictors of dropout and recurrence after liver transplantation for hepatocellular cancer. Transpl Int 2014;27:32-41.

17. Sarraf KM, Belcher E, Raevsky E, et al. Neutrophil/ lymphocyte ratio and its association with survival after complete resection in non-small cell lung cancer. J Thorac Cardiovasc Surg 2009;137:425-8.

18. Liang W, Ferrara N. The Complex Role of Neutrophils in Tumor Angiogenesis and Metastasis. Cancer Immunol Res 2016;4:83-91.

19. Johnson ME, Zhu F, Li T, et al. Absolute lymphocyte count: a potential prognostic factor for Merkel cell carcinoma. J Am Acad Dermatol 2014;70:1028-35.

20. Blomgren H, Lax I, Näslund I, et al. Stereotactic high dose fraction radiation therapy of extracranial tumors using an accelerator. Clinical experience of the first thirty-one patients. Acta Oncol 1995;34:861-70.

21. Ahmed KA, Caudell JJ, El-Haddad G, et al. Radiosensitivity Differences Between Liver Metastases Based on Primary Histology Suggest Implications for Clinical Outcomes After Stereotactic Body Radiation Therapy. Int J Radiat Oncol Biol Phys 2016;95:1399-404.

22. Clark AM, Ma B, Taylor DL, et al. Liver metastases: Microenvironments and ex-vivo models. Exp Biol Med (Maywood) 2016;241:1639-52.

23. Kim HS, Ku JH. Systemic Inflammatory Response Based on Neutrophil-to-Lymphocyte Ratio as a Prognostic Marker in Bladder Cancer. Dis Markers 2016;2016:8345286.

Cite this article as: Xie X, Shan J, Zhou X, Liu H, Sun X. Neutrophil-lymphocyte ratio as a prognostic factor for stereotactic body radiotherapy treatment of metastatic liver tumors. Transl Cancer Res 2020;9(9):5566-5573. doi: 10.21037/tcr20-1335a 